\title{
Semi-analytical approaches to vibrations induced by moving loads
}

\author{
Zuzana Dimitrovová ${ }^{1,2 *}$ \\ ${ }^{1}$ DEC, Faculdade de Ciências e Tecnologia, Universidade Nova de Lisboa, Lisbon, Portugal \\ ${ }^{2}$ IDMEC, Instituto Superior Técnico, Universidade de Lisboa, Lisbon, Portugal
}

\begin{abstract}
With the evolution of the computational power, there is a tendency to overlook analytical and semi-analytical solutions, despite their inherent obvious advantages. One should, however, be aware of the fact, that these solutions provide the necessary insight into the relevant physical phenomena and are accompanied by highly precise results, quickly obtainable without the necessity of additional numerical convergence tests. The objective of this contribution is to fill the gap in available semianalytical solutions related to wave propagation induced by moving loads, with practical applications of high-speed rails. The structures that will be considered are composed of a beam and a supporting medium. The beam represents the interface between the structure and the moving object and will be simplified in conformity with the Euler-Bernoulli theory. In this paper the supporting structure will be considered as a two-parameter viscoelastic foundation and the moving object will be simplified by masses carrying constant forces with harmonic components, under assumption of tight contact. Special attention is paid to the proximity of moving masses.
\end{abstract}

\section{Introduction}

Moving load problems have several practical applications. Nevertheless, in the terminology generally used, it is not always clearly distinguished, whether inertial properties of the moving load are considered or not. This separation is essential, not only because of different dynamic effects that are activated, but also because of the fact that (even in the linear case) solutions related to moving inertial objects cannot be superposed, contrary to the ones related to moving forces.

Thus, moving force problem is far more simple, and in view of this, analytical solutions are available for force traversing finite or infinite beams on linear viscoelastic twoparameter foundation. As already mentioned, it is possible to analyse only one moving force and use superposition, to obtain the desired result. The only issue to deal with is the critical velocity, marking a resonance-type phenomenon, leading to unbounded displacements on infinite beams for one single velocity in the absence of damping. Same phenomenon is present when inertial effects are introduced in the foundation, making the model more realistic, as wave propagation in the foundation is allowed. Superposition is

\footnotetext{
* Corresponding author: zdim@,fct.unl.pt
} 
still possible and critical velocity is then dependent on the mass ratio, defined as the square root of the ratio of the foundation mass to the beam mass, [1-2].

When inertial effects are added to the moving object, then even in the simplest case of one mass moving on a simply supported finite beam without elastic foundation, there is no fully analytical solution. When infinite beam is considered, then essential difference is introduced by the fact whether steady-state solution is searched for, or the movement of the mass in considered from the very initial instant. When steady-state solution is in focus, then inertial effects are removed as time dependent terms. This essential difference is also not always clearly distinguished in the literature. In many cases double Fourier transform, or similar techniques are used, and results obtained are presented as full solution, while they only correspond to the steady-state case. One may argue that the transient vibrations are not important, but this is not true. Without them vibrations would never get unstable, because steady-state solution is reduced to dynamics originated by moving force and in such a case instability has no meaning. When the effect of the harmonic force is added, then the mass value will appear in the final form, but affects only the amplitude value, because the frequency of the resulting vibrations is equal to the excitation frequency.

Analyses of vibrations with emphasis on the initial instant are presented in [3-5]. In these cases, the critical velocity and instability must be clearly distinguished as they are corresponding to fundamentally different phenomena. The critical velocity maintains all its properties and could be determined from the steady-state part of the solution. Instability means exponential increase in amplitudes of the vibrations, which occur for velocities belonging to a certain interval. In fact, instability only occur in supercritical stage. Solution of the problem presented in [3-5] is not fully analytical, its semi-analytical form is based on contour integration, but its main part can be covered by analytical formulas. Beam deflection shape is obtained as a sum of several terms and as important parameters, mass induced frequencies must be determined. The fact that the solution is obtained as a sum of several terms does not mean that we can superpose two or more moving masses. This is immediately seen from the governing equation. In this paper deflection shapes related to two moving masses will be derived and the effect of their proximity will be discussed.

\section{Solution on finite beams}

The governing equation of the problem reads

$$
E I w_{, x x x x}(x, t)+\left(N-k_{p}\right) w_{, x x}(x, t)+m w_{, t t}(x, t)+c w_{, t}(x, t)+k w(x, t)=p(x, t)
$$

where $E I, m$, and $N$ stand for the bending stiffness and mass per unit length of the beam, and a normal force acting on the beam normal axis. $k, k_{p}$ and $c$ are Winkler's and Pasternak's moduli of the foundation and the coefficient of viscous damping of the foundation. Derivatives are designated by the respective variable in subscript position, preceded by a comma. The loading term $p(x, t)$ for two moving masses is

$$
\begin{aligned}
& p(x, t)=\left(P_{1}+P_{01} \sin \left(\omega_{f 1} t+\varphi_{f 1}\right)-M_{1} w_{01, t t}(t)\right) \delta\left(x-x_{1}\right) \\
& +\left(P_{2}+P_{02} \sin \left(\omega_{f 2} t+\varphi_{f 2}\right)-M_{2} w_{02, t t}(t)\right) \delta\left(x-x_{2}\right)
\end{aligned}
$$

where symbols $M, P$ and $P_{0}$ are used for mass, force and amplitude of the harmonic force, respectively. Indexes distinguish the first (rear) and the second (ahead) loading term, $\delta$ is the Dirac delta function, marking location of the load at positions $x_{1}$ and $x_{2}$. As rigid contact is assumed, then 


$$
w_{01}(t)=w\left(x_{1}, t\right)=w(v t, t), w_{02}(t)=w\left(x_{2}, t\right)=w(d+v t, t)
$$

where $d$ is the distance between the two loads and $v$ is the velocity. By application of the chain rule, the loading term updates to

$$
\begin{aligned}
& \left(P_{1}+P_{01} \sin \left(\omega_{f 1} t+\varphi_{f 1}\right)-M_{1}\left(w_{, t t}(x, t)+2 v w_{, x t}(x, t)+v^{2} w_{, x x}(x, t)\right)\right) \delta(x-v t) \\
& +\left(P_{2}+P_{02} \sin \left(\omega_{f 2} t+\varphi_{f 2}\right)-M_{2}\left(w_{, t t}(x, t)+2 v w_{, x t}(x, t)+v^{2} w_{, x x}(x, t)\right)\right) \delta(x-v t-d)
\end{aligned}
$$

Adding convenient boundary and initial conditions, solution can be obtained by eigenmode expansion technique. Equations in modal space are coupled and should be solved numerically. In a matrix form they read

$$
\mathbf{M}(t) \cdot \mathbf{q}_{, t}(t)+\mathbf{C}(t) \cdot \mathbf{q}_{, t}(t)+\mathbf{K}(t) \cdot \mathbf{q}(t)=\mathbf{p}(t)
$$

and individual terms for normalized mode shapes $w_{j}(x)$ are given by

$$
\begin{gathered}
M_{j k}=\delta_{j k}+M_{1} w_{j}(v t) w_{k}(v t)+M_{2} w_{j}(v t+d) w_{k}(v t+d) \\
C_{j k}=\delta_{j k} \frac{c}{m}+2 M_{u 1} v w_{j}(v t) w_{k, x}(v t)+2 M_{u 2} v w_{j}(v t+d) w_{k, x}(v t+d) \\
K_{j k}=\delta_{j k} \omega_{j}^{2}+M_{u 1} v^{2} w_{j}(v t) w_{k, x x}(v t)+M_{u 2} v^{2} w_{j}(v t+d) w_{k, x x}(v t+d) \\
p_{j}=\left(P_{1}+P_{01} \sin \left(\omega_{f 1} t+\varphi_{f 1}\right)\right) w_{j}(v t)+\left(P_{2}+P_{02} \sin \left(\omega_{f 2} t+\varphi_{f 2}\right)\right) w_{j}(v t+d)
\end{gathered}
$$

which does not imply any discretization, but rather involve analytical forms of the mode shapes. As proven in $[3,5]$, such solution can replace solution on an infinite beam. For this, the effect of the supports should be eliminated by application of the load a little further from the support, and then the most convenient supports, can be used, which for such analyses are clearly simple supports.

\section{Solution on infinite beams}

The governing equation in dimensionless variables and parameters, and in moving coordinate $s=x-v t$ is written as

$$
\begin{aligned}
\widehat{w}_{, \xi \xi \xi \xi}+ & 4\left(\eta_{N}-\eta_{S}+\alpha^{2}\right) \widehat{w}_{, \xi \xi}+4 \widehat{w}_{, \tau \tau}-8 \alpha \widehat{w}_{, \xi \tau}+8 \eta_{b}\left(\widehat{w}_{, \tau}-\alpha \widehat{w}_{, \xi}\right)+4 \widehat{w} \\
= & 4\left(2 \eta_{P_{1}}+2 \eta_{P_{01}} \mathrm{e}^{\mathrm{i}\left(\hat{\omega}_{f 1} \tau+\varphi_{f 1}+3 \pi / 2\right)}-\eta_{M_{1}} \widehat{w}_{, \tau \tau}\right) \delta(\xi) \\
+ & 4\left(2 \eta_{P_{2}}+2 \eta_{P_{02}} \mathrm{e}^{\mathrm{i}\left(\hat{\omega}_{f 2} \tau+\varphi_{f 2}+3 \pi / 2\right)}-\eta_{M_{2}} \widehat{w}_{, \tau \tau}\right) \delta(\xi-\widehat{d})
\end{aligned}
$$

where the set of parameters reads

$$
\begin{gathered}
\chi=\sqrt[4]{\frac{k}{4 E I}}, \xi=\chi s, v_{c r}=\sqrt[4]{\frac{4 k E I}{m^{2}}}=\frac{1}{\chi} \sqrt{\frac{k}{m}}, \tau=\chi v_{c r} t, \widehat{w}=\frac{w}{w_{s t}}, \widehat{\omega}_{f j}=\frac{\omega_{f j}}{\chi v_{c r}} \\
w_{s t}=\frac{P_{1} \chi}{2 k}, \eta_{P_{j}}=\frac{P_{j}}{P_{1}}, \eta_{P_{0 j}}=\frac{P_{0 j}}{P_{1}}, \eta_{M_{j}}=\frac{M_{j} \chi}{m}
\end{gathered}
$$




$$
\alpha=\frac{v}{v_{c r}}, \eta_{b}=\frac{\mathrm{c}}{2 \sqrt{m k}}, \eta_{N}=\frac{N}{2 \sqrt{k E I}}, \eta_{S}=\frac{k_{p}}{2 \sqrt{k E I}}
$$

When applicable $j=1,2$; and $\hat{d}$ is the dimensionless counterpart of the distance between the loads.

After specification of boundary and initial conditions, solution steps involve application of Laplace and Fourier transforms. Then the inverse Fourier transform can be performed analytically, leading to the analytical expression for the Laplace image of the beam deflection shape, and finally, the inverse Laplace transform can be obtained by contour integration. Final solution is expressed as a sum of several terms (sum of residues), with fully analytical expressions except for the induced frequencies that have to be obtained numerically. As proven in [5], unfortunately, there is a discontinuity in one of the involved functions on the complex $q$-plane and thus the previous expressions must be accompanied by a term coming from the numerical integration along the branch cuts. This function is defined as

$$
\begin{gathered}
K(\xi, q)=\int_{-\infty}^{\infty} \frac{\mathrm{e}^{\mathrm{i} \xi p} \mathrm{~d} p}{D(p, q)} \\
D(p, q)=p^{4}-4 p^{2}\left(\eta_{N}-\eta_{S}+\alpha^{2}\right)-4 q^{2}+8 \alpha p q+8 \mathrm{i} \eta_{b}(q-\alpha p)+4
\end{gathered}
$$

which can also be obtained as a sum of residues.

Nevertheless, it has been shown that vibrations resulting from the integration along the branch cuts, are important for adapting the solution to the initial conditions, but generally can be neglected in most cases. Previous expression coming from the inverse Laplace transform is just sufficiently accurate, [3-5]. In the case of two masses, the induced frequencies are computed as complex roots of

$$
\left(\pi-2 \eta_{M_{u 1}} q^{2} K(0, q)\right)\left(\pi-2 \eta_{M_{u 2}} q^{2} K(0, q)\right)-4 \eta_{M_{u 1}} \eta_{M_{u 2}} q^{4} K(\hat{d}, q) K(-\hat{d}, q)
$$

where the last term expresses the coupling between the two masses, because for one moving mass, analogous expression is given by, [3]:

$$
\pi-2 \eta_{M_{u 1}} q^{2} K(0, q)
$$

The induced frequencies as roots of Eq. (15) can be again obtained by iteration techniques described in [4-5]. Now, more attention must be paid to the individual iterations, as generally, there are more roots because the expression in Eq. (15) is of higher order than in Eq. (16).

\section{Results}

As an example, the case with input data given in [3], where it is designated as Case 1, is used. Vibrations induced by two masses of equal value carrying constant forces of equal value without harmonic component are analysed. The effect of their distance is examined using the values of induced frequencies and displacements of the contact point of the rear mass. As already mentioned, superposition cannot be used, but when the masses are sufficiently apart, the difference between the approximate solution obtained by superposition and correct solution is not significant. When masses are getting closer, the error increases and for closely located masses it would be better to use a single load of 
doubled mass and force. This can be simply demonstrated on the following Figure 1. The orange curve represents displacement of the contact point of one moving mass. The grey line corresponds to the case when the mass and force are doubled, but for the sake of comparison, same static displacement of one single force is used. As expected, it is seen that the induced frequency is quite different, so is the amplitude. Blue line represents the correct solution and the red one the approximate solution obtained by superposition. Separate parts of the figure shown situations for different mass distances.
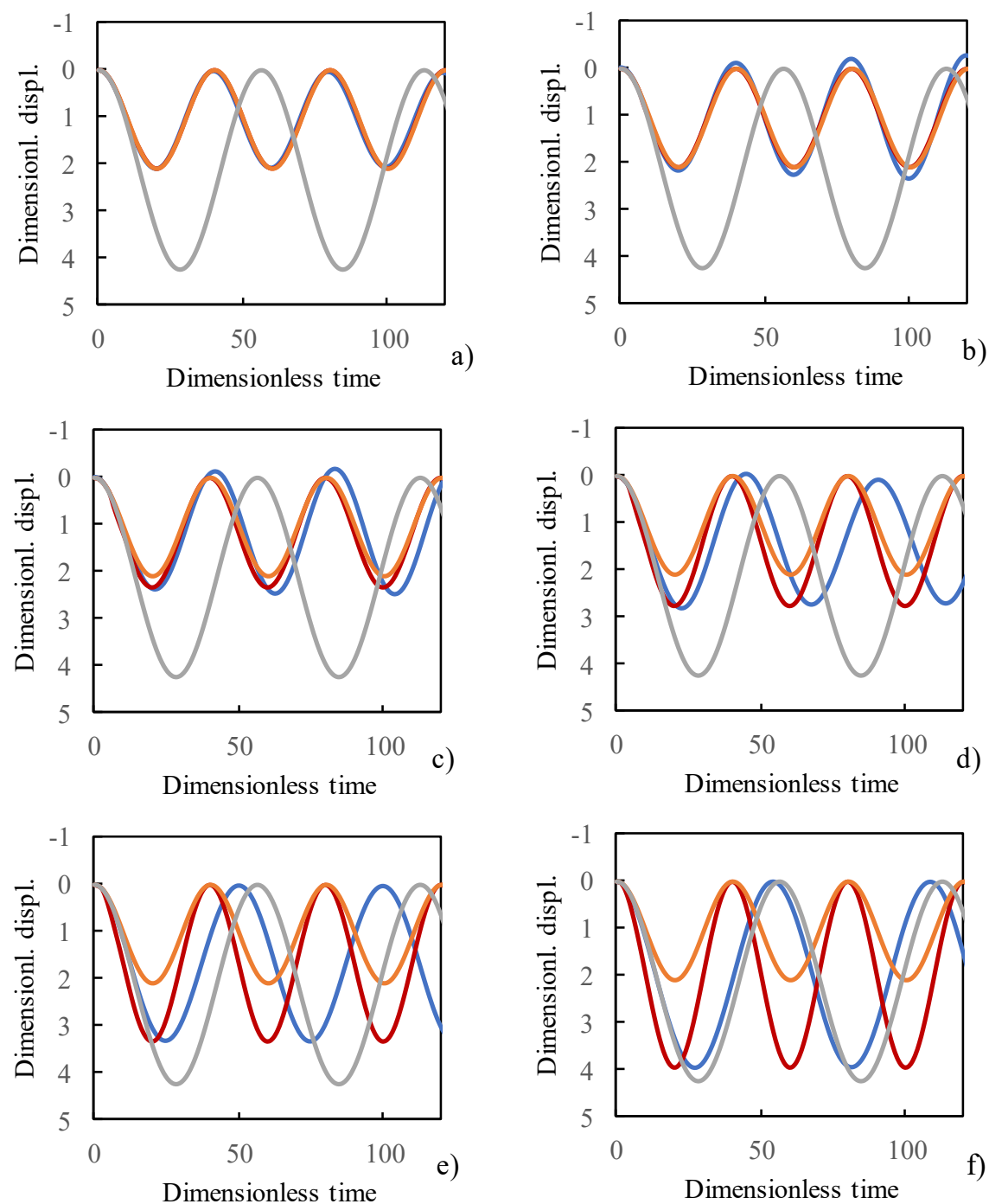

Figure 1. Displacement of the contact point of the rear mass for different distances between the two masses, expressed as multiple of $\chi$ : a) 10 ; b) 5 ; c) 4 ; d) 3 ; e) 2 ; f) 1 .

It is seen in Figure 1, that the superposition case naturally maintains the induced frequency as for one moving mass, but the correct solution is changing the induced frequency, gradually approaching the value for one double mass with decreasing distance between the masses. By defining a convenient norm, it is possible to establish acceptable masses distance for which superposition can still be used and another one, when better solution 
would be obtained by joining masses. Due to the analytical expressions, this is a simple and quick task.

Same conclusion can be obtained by analysing induced frequencies. There are in fact two frequency lines for most velocities in subcritical region (Figure 2a)). One is lower, and the other one is higher than the frequency line of one moving mass, which is in fact not shown on the same figure for the sake of clarity. The higher line is interrupted by the cutting frequency. As no damping was assumed, each point on frequency line corresponds to one and two pairs of frequencies, depending whether is located in subcritical or supercritical region. Without damping, the onset of instability is kept at the critical velocity. In supercritical region, frequency lines are not so smooth as for one moving mass, as demonstrated in Figure 2b). Grey line marks the cutting frequency.
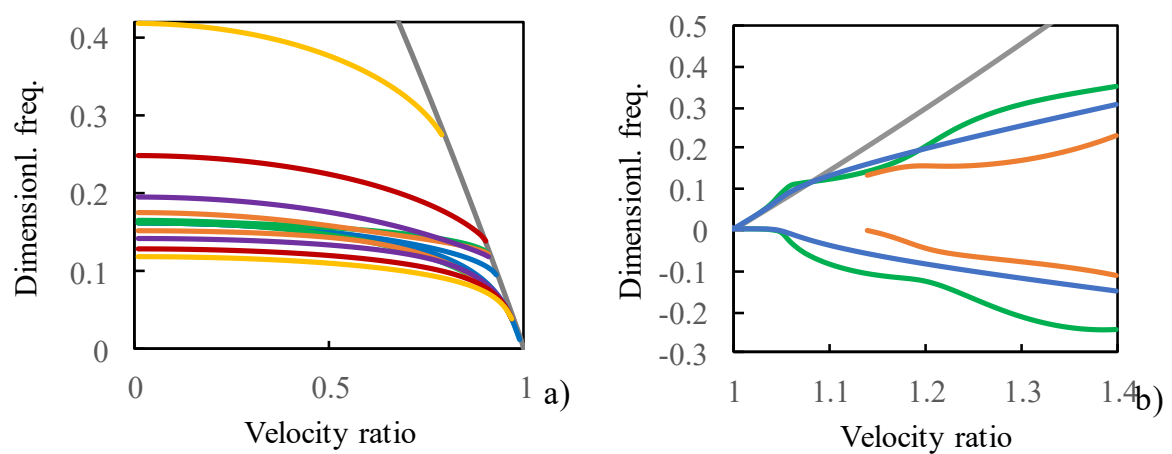

Figure 2. a) Frequency lines as a function of the distance between the masses (two masses distant by multiple of $\chi$ : 10 - blue; 5 - green, 4 - orange, 3 - violet, 2 - red, 1 - yellow); b) frequency lines in supercritical range (one mass - blue; two masses distant by $10 \chi$ : green and orange).

\section{Conclusions}

In this paper it was shown that by using semi-analytical solutions, several analyses on the quality of the vibrations induced by moving loads can be performed quickly and accurately without testing numerical convergence of discretized models. This way, it is possible to obtain straightforwardly accurate results in dimensionless forms, and thus encompassing in few graphs results related to all possible parameter combinations. As an example, analysis of the influence of the proximity of moving masses was selected. Quite obvious conclusion was confirmed: when masses are sufficiently apart, the error introduced by results superposition is negligible, while when masses are very close, it would be better to replace them by one load with doubled mass and force. In addition, it was shown that the instability region is not affected in an undamped case and the onset of instability occurs exactly at the critical velocity, as for one moving mass.

This work was supported by FCT, through IDMEC, under LAETA, project UID/EMS/50022/2013.

\section{References}

[1] Z. Dimitrovová, J Sound Vib 366, 325-342 (2016)

[2] Z. Dimitrovová, Int J Solids Struct 122-123, 128-147 (2017)

[3] Z. Dimitrovová, Int J Mech Sci 127, 142-162 (2017)

[4] Z. Dimitrovová, Int J Mech Sci 144, 283-311 (2018)

[5] Z. Dimitrovová, J Sound Vib, in press (2018) 